\title{
Determination of the fluorescence spectrum of Botrytis cinerea Pers.:Fr. isolated from highbush blueberry (Vaccinium corymbosum L.)
}

\author{
M. Cerna Larenas ${ }^{1,4^{*}}$, R. Guzmán Estrada ${ }^{1,4}$, J. Guerrero Contreras ${ }^{2}$, C. Meriño \\ Gergichevich $^{3}$ \\ Departamento de Ciencias Físicas, Facultad de Ingeniería y Ciencias. Universidad de La Frontera, Temuco, \\ Chile. Departamento de Producción Agropecuaria, Facultad de Ciencias Agropecuarias y Forestales. \\ Universidad de La Frontera, Temuco, Chile. Center of Plant, Soil Interaction and Natural Resources \\ Biotechnology, Scientific and Technological Bioresource Nucleus (BIOREN-UFRO), Universidad de La \\ Frontera, Temuco, Chile. Center for Optics and Photonics, Universidad de Concepción, Concepción, Chile. \\ *Corresponding author: manuel.cerna@ufrontera.cl
}

\begin{abstract}
The prompt diagnosis of an attack by phytopathogens associated with the deterioration of the quality and condition of highbush blueberry is of great interest, particularly when dealing with Botrytis cinerea in preand postharvest stages. Real-time technologies that complement traditional diagnostic methods during the processing, storage and transport of fruit are advisable. The aim of this study was to determine the fluorescence spectrum of the $B$. cinerea mycelium in culture medium as well as its temporal variation. Two evaluations were conducted daily for 18 days using a fluorimeter with a $100 \mathrm{~mW}, 405 \mathrm{~nm}$ laser light, which excited the mycelium of each of five isolates of $B$. cinerea in two fixed positions. The fluorescence emission of the malt agar culture medium and the emission obtained from the fungus Trichothecium roseum were quantified for comparative reference. It was found that $B$. cinerea fluoresces mainly in the red-infrared area of the spectrum and $T$. roseum does so with greater intensity in the green-red segment. The energy of the fluorescence spectrum did not vary significantly according to the development of the $B$. cinerea mycelium in artificial culture medium.
\end{abstract}

Keywords: Botrytis cinerea, fluorescence spectrum, highbush blueberry

\section{Introduction}

Fluorescence spectroscopy is a technique that analyzes the fluorescence of a solid or liquid sample using generally blue or ultraviolet light as the excitation signal. Determination of the wavelengths and intensities of the fluorescence spectrum provides important information on the structure of the vibration levels of the constituent molecules. The device used for fluorescence spectroscopy is called 
a fluorimeter or fluorometer and essentially consists of a light source with its corresponding filter for the selection of the wavelength excitation, a sample, a filter to separate the signal that the sample emits, and a sufficiently sensitive optical detector. Fluorimetry applications include chemical, biochemical and medical analyses, high-energy physics, nuclear physics, diagnosis through medical imaging, identification of microscopic biological species, and marking of reagents and antibodies (Rubinson and Rubinson, 2001; Skoog, 2001).

Fluorescence spectroscopy has comparative advantages over similar techniques available such as infrared spectroscopy, Raman spectroscopy and Coherent Anti-Stokes Raman Scattering (CARS) spectroscopy. Indeed, the intensity of the fluorescence signal from plant samples can be detected more simply and affordably; the fluorescence excitation signal can be selected from ultraviolet to red, depending on the sample, although ultraviolet radiation (UV) is the most common. The optical system of a typical fluorimeter is smaller, cheaper and more robust; the mechanical adjustment of the various optoelectronic elements is simpler and has higher tolerance. In this sense, simplicity in detection is a great advantage when implementing a system that can function in the field and without the need of an expert (Withnall, 2003; Pereira, 2010; Qin, 2011; Soler, 2013; Mota et al., 2014).

It should be noted that plant diseases inflict damage worldwide with huge production and economic losses in the agricultural and forestry industries. The main causes are fungi, bacteria and viruses (Sankaran, 2010). Currently, molecular techniques to identify phytopathogens are used frequently, requiring the meticulous process of sample taking and data processing. Early and reliable information on the health of the culture through a precise and prompt diagnosis would contribute to efficient preharvest and postharvest disease control (Pérez et al., 2014; Porep et al., 2015). The methods generally used to detect diseases in plants and trees can accurately identify the organisms causing the disease, either directly or indirectly. This latter case uses fluorescent or chromatic markers to characterize the biophysical and chemical aspects of the host. The infectious biotic cause of a disease can be confirmed using accurate but slow and elaborate techniques such as Enzyme-Linked InmunoSorbent Assay (ELISA) and Polymerase Chain Reaction (PCR). Nevertheless, it is also possible to detect disease at the onset of pathogenesis using other techniques that are sufficiently accurate, rapid and feasible to apply at specific sites and in real time (Sankaran, 2010; Pourreza et al., 2015).

In recent decades, laser-induced fluorescence has been used to determine stress levels in plants (Belasque, 2008). Thus, for example, it has been established that the fluorescence of chlorophyll is a precise and nondestructive indicator of the photochemical efficiency of the photosynthetic apparatus, reflecting the impact of different environmental factors (Cerovic, 1999; Maxwell and Johnson, 2000; Cuchiara et al., 2015). When the spectrum is broadened to the near-infrared (700-1200 nm), the spectral properties of the leaf mesophyll cell structure can be determined, and in the absorption range of the mid-infrared (1200-2400 nm) correlations have been shown with the water content of the plant (Usha, 2013). In this context, Belasque et al. (2008) used fluorescence spectroscopy to detect the stress caused by the bacterial disease citrus canker using a portable spectrometer with laser excitation (Nd:YAG $2^{\text {nd }}$ harmonic of $532 \mathrm{~nm}$ and $10 \mathrm{~mW}$ (miliwatt). They obtained the differentiated fluorescence spectrum 
for the causalities being studied. Lins et al. (2009) detected an attack of citric canker at a site in Brazil. These studies exposed the possibility of diagnosing diseases in citrus cultures through the spectral analysis of the leaves. Additionally, florescence spectroscopy with incorporated imaging can detect the Tobacco Mosaic Virus (TMV) after $50 \mathrm{~h}$ of inoculation, as well as yellow rust (Puccinia striiformis) in plants at a 96\% certainty (Bravo et al., 2004).

In Chile, $B$. cinerea is one of the main diseases in highbush blueberries. This organism causes rot postharvest, and the phenological stages with the highest susceptibility are flowering, fruit set and ripening (Guerrero, 1988; Pino, 1992; Belmar, 1993; Bensch, 1996). The incidence of B. cinerea postharvest varies depending on the cultivar, favorable environmental conditions, and pre- and postharvest crop management. In this respect, Pino (1992) and Bello (1992) presented postharvest values between $1 \%$ and $16 \%$ depending on the cultivar. Thus, it is prudent to detect and identify phytopathogens through the use of technologies such as infrared fluorescence spectroscopy, absorption spectroscopy and others that can be implemented in the field and can generate information in real time during the processing, storage and transport of blueberries.

This study is the first stage of a process that endeavors to develop a detection system for the early or developed infection of $B$. cinerea in blueberry fruits. It is ultimately expected that the information generated by this system will support the technical decision-making involved in the commercialization of this fruit. The aim of this study was to determine the spectral fluorescence signature of $B$. cinerea in vitro under laboratory conditions. Additionally, the temporal evolution of the fluorescence spectrum was assessed and the fluorescence spectral characteristics of $B$. cinerea were contrasted with the fluorescence spectrum of Trichothecium roseum.

\section{Materials and Methods}

\subsection{Assembly of the fluorimeter}

Using a Thorlabs PTM11113 optical table, a conventional fluorimeter was configured using a 700 $\mathrm{mW}, 405 \mathrm{~nm}$, S06J single mode blue laser diode with a $7 \mathrm{~nm}$ emission bandwidth. Both the temperature and the supply current of the laser diode were controlled using specially designed electronic systems. The laser beam affects the sample by generating its fluorescence (Figure 1); the emitted signal, with a longer wavelength than the incident signal, is reflected in the dichroic mirror (DM), collected with a converging lens and quantified by a detector. Because the DM does not reflect the blue light originating from the laser, it is possible to separate the intense excitation signal from the weak emission signal, thereby avoiding saturation of the sensor.

The diode laser (DL) along with its collimator lens (CL), the iris diaphragm, the DM and the sample are located on the same vertical line. This setup has the great advantage of not requiring the use of a cuvette to hold the sample. Moreover, the vertical position of the sample is not critical and is almost independent of the position on the horizontal plane; therefore, high precision in the position of the base that supports the sample is not necessary to obtain consistent spectral measurements. The iris diaphragm allows control of spurious light and unwanted reflections. 


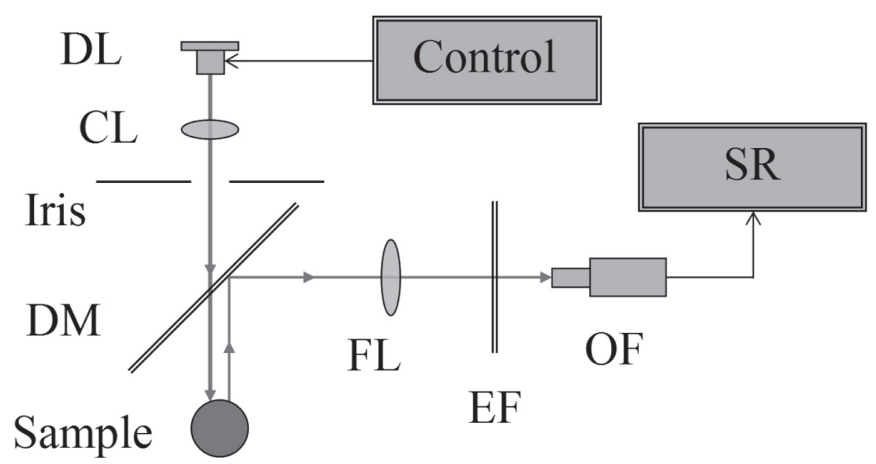

Figure 1. Diagram of the fluorimeter used. DL: diode laser, CL: collimator lens, DM: dichroic mirror, FL: focusing lens, EF: emission filter, OF: optical fiber, SR: spectroradiometer detector. The excitation signal originating from the laser causes the sample to emit a fluorescence signal that is quantified by the detector (adapted from Skoog, 2001).

\subsection{Treatments}

The four treatments included in this study were: Dish A: Malt Agar (MA) culture medium used as the control (Figure 2.A), Dish B: Isolate of B. cinerea in MA culture medium (Figure 2.B), Dish R: MA culture medium without the $B$. cinerea mycelium (Figure 2.R), Dish T: isolate of Trichothecium roseum (Figure 2.T).

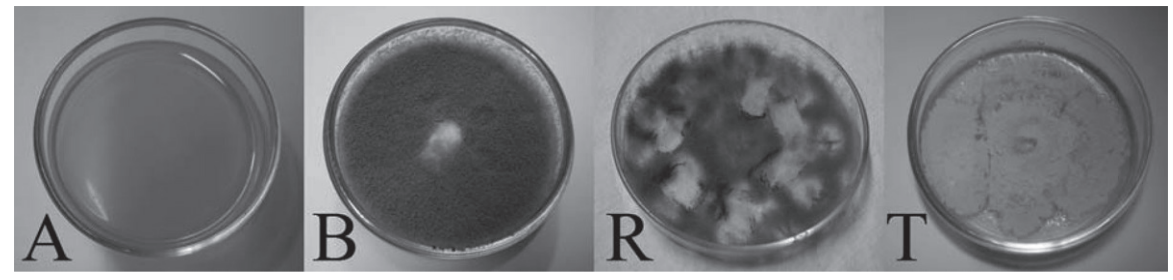

Figure 2. Treatments considered, (A) Dish A: MA culture medium, (B) Dish B: isolate of Botrytis cinerea, (R) Dish R: scraped MA culture medium, (T) Dish T: Trichothecium roseum. Phytopathology Laboratory, Universidad de La Frontera.

\subsection{Preparation of the treatments}

The $\mathrm{R}$ Dishes were obtained from isolates of $B$. cinerea that were cleaned mechanically prior to each measurement by scraping the mycelium of the fungus with a small sterile wooden spatula. Thus the spectrum could be obtained from the MA medium and the effect of the fungus on the substrate could be specified. T. roseum (Dish T) was used as a contrast for $B$. cinerea (Dish B) to assess the discrimination potential between the two through the use of the fluorescence spectrum obtained. It is possible to clean $\mathrm{R}$ Capsules chemically; however, these substances could affect the development of $B$. cinerea. As a result, 
mechanical cleaning was performed, taking special care not to damage the fragile surface of the culture medium. The B Dishes of B. cinerea were incubated in the Phytopathology Laboratory in the Agroindustrial Institute of the Universidad de La Frontera, maintaining them in a culture chamber for $96 \mathrm{~h}$ at 25 ${ }^{\circ} \mathrm{C}$ and $80 \%$ humidity. The T. roseum isolates used were dated as being at 30 days of development. The evaluation period using the fluorimeter was 18 days under laboratory conditions of natural light and room temperature $\left(20 \pm 2{ }^{\circ} \mathrm{C}\right)$.

In preliminary assays, it was observed that the pure MA culture medium and the $T$. roseum presented a consistent and stable fluorescence spectrum over time; therefore, in this study the samples corresponding to these categories were not studied daily (in contrast to the $B$. cinerea). A complete series of measurements was conducted over 18 consecutive days. The B Dishes were measured daily from days 1 to 18 ; however, Plates R, A, and T were measured only on days 2, 11 and 18. When the daily measurement was made for any category, each Petri Dish was always radiated in the same position and with the same optical power so that a follow-up of the fluorescence spectrum could be generated in the same location where the fungus developed. Each measurement was taken in duplicate in each Petri Dish, which was considered to be the experimental unit. The detector was a LI-1800 portable spectroradiometer (LICOR INC, USA), which collected the fluorescence emission signal by means of an optical fiber. Each measurement using the equipment integrated three consecutively acquired spectra, a process which took approximately two minutes. The total time needed for the daily recording of the spectra only in the category B Capsule (Botrytis), considering five samples, two areas measured per sample and three integrated signals for each measured area, was approximately one hour. The laser was controlled accurately using the electronic systems, and its stability was verified by means of $75-\mathrm{g} \mathrm{cm}^{-2}$ standard white alkaline paper, which was used as the reference sample of the optical excitation power. Data processing was performed in Math Works Matlab v. 7.6.0.324 (R2008a). The differences between the fluorescence spectra of the Dish B and Dish T samples were evaluated using Student's t-test of independent data.

\section{Results}

\subsection{Fluorescence spectrum of the culture medium (Dish A)}

The fluorescence spectra of five replicates of the Dishes in each treatment showed strong similarity, making their accurate representation possible using their mean value curve. The fluorescence emission of the mechanically stripped culture medium of the fungus (category R Dish) and the mean fluorescence curve of the pure MA substrate (category A Dish) are compared in Figure 3. Although the intensity of the standard fluorescence is greater in the green area of the spectrum $(500-570 \mathrm{~nm})$, the emission extends across the entire visible range.

The curves in Figure 3 reveal that the growth of B. cinerea on MA (Dish R) only slightly affected the fluorescence emission of the culture medium, particularly in the 600 to $700 \mathrm{~nm}$ range. This slight effect can be explained by the possibility that during the scraping process, the surface of the culture medium had not been cleaned completely; leaving traces invisible to the naked eye that changed the optical characteristics of the medium. It is also likely that the growth structures of the fungus itself had penetrated the substrate and caused this result. Because the observed effect was small and limited solely to certain wavelengths, it is possible to disregard it and use the average substrate spectrum obtained from the 
pure malt agar samples (Dish A) as the reference spectrum. In this way, future studies can avoid the difficulty of obtaining the reference spectrum from fungal samples that must be cleaned frequently during the series of measurements to be taken.

\subsection{Typical fluorescence spectra of Dish B and Dish $T$}

An example of standard fluorescence spectra obtained directly from the samples in Dish B (B. cinerea) and Dish T (T. roseum), together with the pure MA average is illustrated in Figure 4. The underlying general shape of the fungal spectra is due to the fluorescence of the MA culture medium. A lower signal-to-noise ratio is observed in Dish B compared with Dish $\mathrm{T}$ because the signal emitted by the former was considerably weaker.

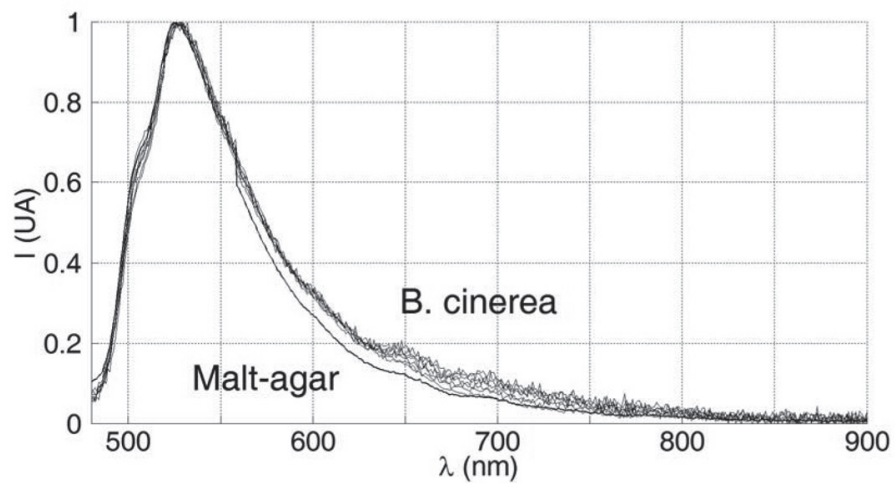

Figure 3. Standard fluorescence of the samples of scraped B. cinerea (Dish R) and the pure MA average obtained from the Dish A samples. A general similarity is observed between the two spectral responses with a marked emission in the green area of the spectrum at approximately $530 \mathrm{~nm}$.

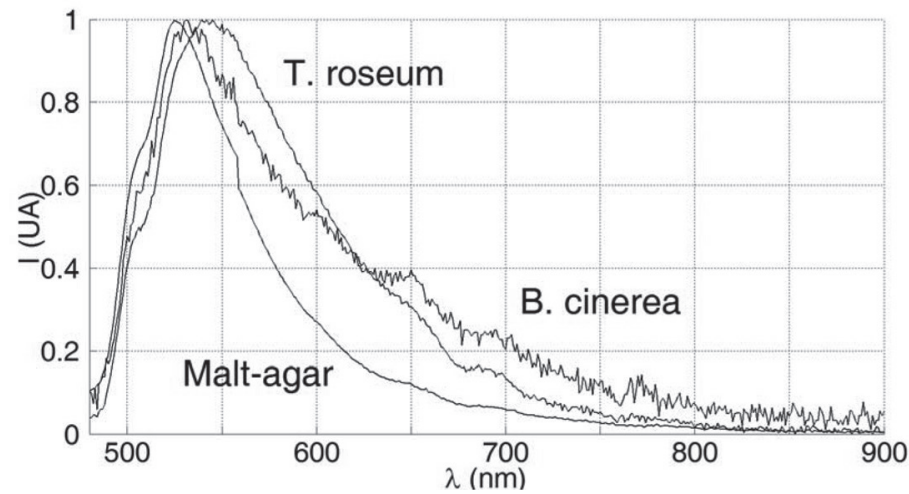

Figure 4. Fluorescence spectra corresponding to daily measurements. The curves shown are the emissions of the MA substrate and the fungi $T$. roseum and $B$. cinerea growing on the MA substrate. 


\subsection{Fluorescence spectra of B. cinerea and T. roseum}

Once the fluorescence curve of the culture medium was determined (pure MA curve), the fluorescence emission curve of $B$. cinerea was derived. To do this, the spectrum obtained from each of the measurements of the category B Dish was subtracted from the reference spectrum emitted by the pure MA culture medium, which allowed for the corresponding adjustment of the intensities. Thus, the fluorescence signal emitted only by the fungus of interest is obtained, eliminating the signal emitted by the underlying MA substrate.

The resulting final spectrum for the Dish B sample set is presented in Figure 5. It can be observed that $B$. cinerea generated fluorescence mainly in the red area of the spectrum (640-760 nm), enabling the efficient spectral separation of the powerful laser excitation signal located at $405 \mathrm{~nm}$. The $T$. roseum spectrum was obtained in a similar fashion as also shown in Figure 5. In contrast to $B$. cinerea, this fungus emitted at a greater intensity primarily in the green-red area of the spectrum (550-640 nm).

To quantify the differences between the fungal spectra, the Disease Index was used, defined as $\mathrm{Fg}=\mathrm{I} 550$ $(\mathrm{I} 550+\mathrm{I} 690)^{-1}$, where I550 and I690 are the fluorescence intensities at 550 and $690 \mathrm{~nm}$, respectively (Figure 6). Next, a t-test for independent samples was conducted to determine the existence of significant differences between the indices of the samples. For $B$. cinerea, the mean of the Disease Index was 0.0080 with a standard deviation of 0.0868 and in the case of T. roseum, a mean of 0.7691 with a standard deviation of 0.0156 was obtained (Figure 6). When comparing the means using a t-test for independent samples, it was found that the difference was highly significant; i.e., the probability of finding such a difference between the means of the Disease Indices of the sampled spectra, when in fact there are no differences in the total population, is extremely low. The results of the $\mathrm{t}$-test for independent samples were: two-tailed $\mathrm{p}$ value $<0.0001$; difference between means: 0.7611 ; and $95 \%$ confidence interval: 0.813 to 0.709 .

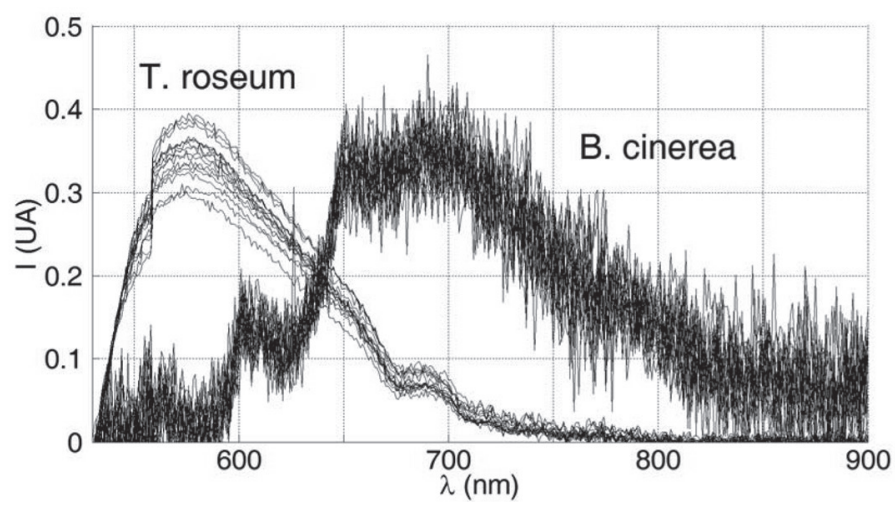

Figure 5. Left: Standard fluorescence emission curves of T. roseum from all the samples, using the pure MA average as reference. Right: Standard fluorescence emission curves of $B$. cinerea from all the samples, using the pure MA average as reference. The curves shown include the five samples of $B$. cinerea and all the days. 


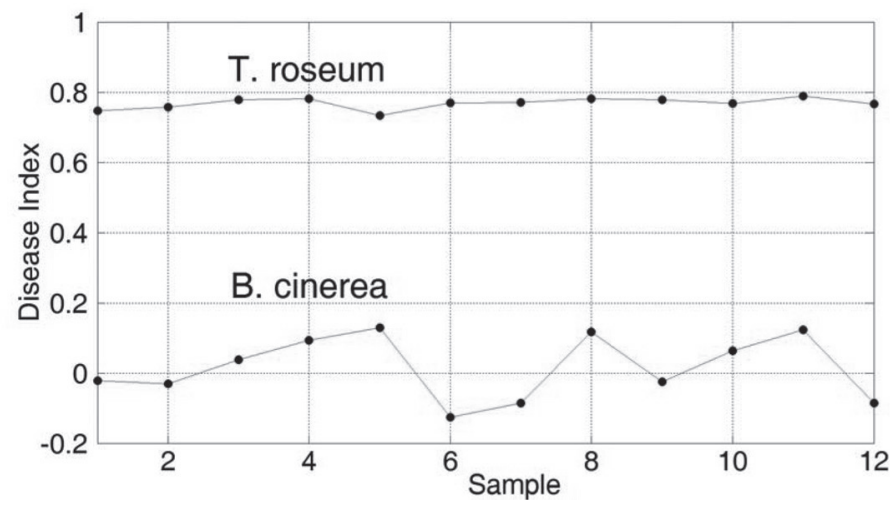

Figure 6. Disease Index values obtained from the fluorescence spectra of samples from both fungi.

\subsection{Evolution of the spectrum of Botrytis cinerea}

The fluorescence spectra for $B$. cinerea are shown in Figure 5. Because these have a very similar general shape, a representative value can be used; for example, the mean value of each of the spectra to follow their evolution as the fungus develops. Accordingly, for each sample, a numerical integration between 550 and $900 \mathrm{~nm}$ was performed for each of the non-standard curves obtained. The resulting values serve as an indicator of total light intensity for each fluorescence spectrum and can be compared to determine whether significant changes are produced as the $B$. cinerea mycelium evolves (Figure 7).

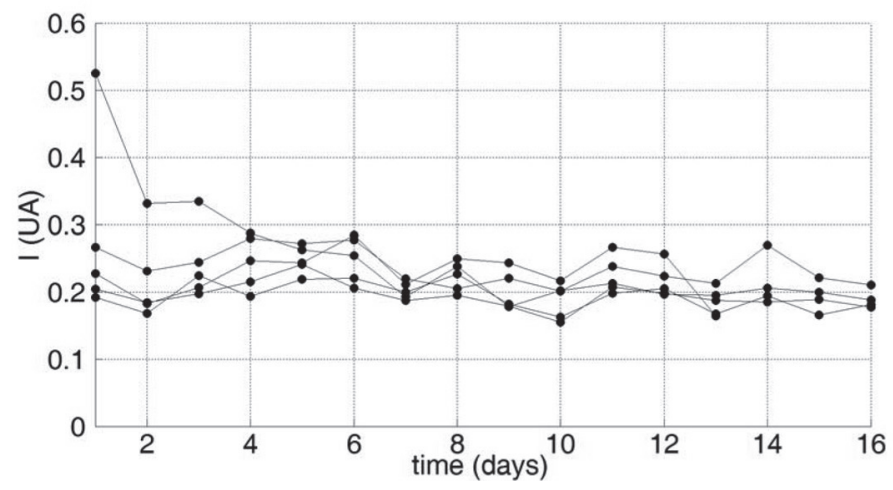

Figure 7. Evolution of the total intensity of the spectrum from each of the five samples of $B$. cinerea. Generally, no significant variation in the values was observed. 


\section{Discussion}

One aspect of special importance when working with biological samples is the maximum energy able to support the organism before possible damage. In the case of the fluorimeter with continuous laser, the light intensity, i.e., the optical power per unit of area was regulated by varying the laser excitation power or modifying the exposure area by the CL (Figure 1). Assuming that the fungi evaluated in this work are able to resist exposure to direct sunlight at noon at the height of summer when the solar constant at ground level reaches approximately $1000 \mathrm{Wm}^{-2}$, and using 50 $\mathrm{mW}$ of optical power in the excitation laser, the area to radiate using the laser under the conditions indicated would be $50 \mathrm{~mm}^{2}$, if the area of interest is circular, the diameter would be $8 \mathrm{~mm}$. Because the fluorescence spectrum of $B$. cinerea is very weak, it is necessary to excite the sample with the greatest possible intensity. By observation under a stereoscopic magnifying glass and optical microscope, the possible damage of the $B$. cinerea mycelium was evaluated when radiated with $50 \mathrm{~mW}$ in a circular area $5 \mathrm{~mm}$ in diameter, in which case the intensity reached $2500 \mathrm{~W} \mathrm{~m}^{-2}$; the spectral differences between sunlight and the blue laser used were not considered in these calculations. In this respect, it is documented that a biological sample can be excited with a very powerful signal but one of very short duration with a pulsed laser used in commercial equipment. The use of a continuous laser, i.e., the system used here, is also viable when the sensor system has a slower response.

Using laser fluorescence spectroscopy, it was possible to determine the characteristic spectrum of $B$. cinerea in advanced stages of growth when the presence of the fungus was observable using the naked eye. The spectral emittance curve (Figure 4) showed that the fungus emitted mainly in the red-infrared area of the spectrum when excited with a $100-\mathrm{mW}$, 405-nm laser. Because the fluorescence spectrum characteristic of another common fungus (Figure 5) was also determined, it may also be concluded that $B$. cinerea can be distinguished from $T$. roseum through its fluorescence spectral signature using the Disease Index as an indicator (Figure 6). The t-test for independent data indicated that the differences between the means of the indices of $B$. cinerea and $T$. roseum were highly significant.

The integrated values are summarized in Figure 7, which shows that except for one of the samples during the first three days, no significant variation was detected in the intensity of the fluorescence spectra while the samples developed in the capsule. The same general behavior was obtained when the integration process was repeated using other wavelength ranges. This finding shows that it would not be possible to distinguish the different developmental stages of the fungus using fluorescence, even less so in the early stages when the fungus is not visible to the naked eye, and when the signal emitted by $B$. cinerea would be undetectable due to masking by the emission of the substrate. Therefore, it may be necessary to evaluate the use of other more refined and/or sensitive spectroscopic techniques to detect this fungus in its early stages.

\section{Conclusions}

The spectral signature and temporal variation of the fluorescence spectrum of the mycelium of the phytopathogenic fungus Botrytis cinerea in malt agar medium under laboratory conditions was determined. The total intensity of the fluorescence emission spectrum did not vary significantly according to the development of the $B$. cinerea mycelium in artificial culture medium. The potential of the fluorescence 
spectrum was demonstrated quantitatively to differentiate the fungi Botrytis cinerea and Trichotecium roseum under laboratory conditions.

\section{Acknowledgements}

The author expresses thanks for the partial support provided by PIA-CONICYT PFB0824 and the DIUFRO through project DI08-0063.

\section{References}

Belasque, J., Gasparoto, M., Marcassa, G. 2008. Detection of mechanical and disease stresses in citrus plants by fluorescence spectroscopy. Appl. Optics. 47, 1922-1926.

Bello, U. 1992. Tratamientos fungicidas de precosecha y comportamiento en almacenaje refrigerado de arándano (Vaccinium corymbosum L.). Tesis Ing. Agr. Universidad de la Frontera. Facultad de Ciencias Agropecuarias. Temuco. Chile 136p.

Belmar, H. 1993. Incidencia del período entre cosecha, prefrío y momento de cosecha en la calidad e infección por hongos de postcosecha, en fruta de arándano alto (Vaccinium corymbosum L., cvs. Elliot y Berkeley). Tesis Ing. Agr. Universidad de La Frontera. Facultad de Ciencias Agropecuarias. Temuco. Chile 114p.

Bensch, E. 1996. Estados fenológicos de mayor susceptibilidad, efecto inhibitorio (DE 50) y efectividad de benomil + captan y BC-1000 para el control de Botrytis cinerea y Alternaria alternata en fruto de arándano alto (Vaccinium corymbosum L.) cv. Bluejay. Tesis Ing. Agr, Universidad de la Frontera. Facultad de Ciencias Agropecuarias. Temuco. Chile. 106 p.
Bravo, C., Moshou, D., Oberti, R., West, J., McCartney, A., Bodria, L., Ramon, H. 2004. Foliar disease detection in the field using optical sensor fusion. Agricultural Engineering International, Vol VI: The CIGR Journal of Scientific Research and Development, Manuscript FP 04 008, Vol. VI.

Cerovic, Z., Samson, G., Morales, F., Tremblay, N., Moya, I. 1999. Ultraviolet-induced fluorescence for plants monitoring: present state and prospects. Agronomie. 19, 543-578.

Cucchiara, C.C., Silva, I.M.C., Dalberto, D.S., Bacarin, M.A., Peters, J.A. 2015. Chlorophyll a fluorescence in sweet potatoes under different copper concentrations. J. Soil Sci. Plant Nutr. 15, 179-189.

Guerrero, J. 1988. Enfermedades del arándano en Chile. En: Seminario El cultivo del arándano. E.E. INIA-Carillanca. Programa Frutales y Viñas. Serie Carillanca $N^{\circ}$ 2. pp 99-108.

Lins, E., Belasque, J., Marcassa, L. 2009. Detection of citrus canker in citrus plants using laser induced fluorescence spectroscopy. Precis. Agric. 10, 319330.

Maxwell, K., Johnson, G. 2000. Chlorophyll fluorescence, a practical guide. J. Exp. Bot. Vol. 51, 659-668.

Mota, A., Rossi, G., Cunha de Castro, G., Ortega, G., de Castro, J. 2014. Portable fluorescence spectroscopy platform for Huanglongbing (HLB) citrus disease in situ detection. Light-Emitting Diodes: Materials, devices, and applications for solid state lighting XVIII. Proc. of SPIE 9003, 90031U.

Pereira, D. 2010. Espectroscopia Raman aplicado ao beta-caroteno. Tesis Mag. Física. Universidade Federal do Pará. Belém. Brasil. 65p. 
Pérez, S., Meriño-Gergichevich, C., Guerrero, J. 2014. Detection of Neofusicoccum nonquaesitum causing dieback and canker in highbush blueberry from Southern Chile. J. Soil Sci. Plant Nutr. 14, $581-588$.

Pino, M. 1992. Comportamiento en almacenaje refrigerado de fruta de once cultivares de arándano alto (Vaccinium corymbosum L.) e incidencia y control de hongos en postcosecha. Universidad de la Frontera. Facultad de Ciencias Agropecuarias. Temuco. Chile. 136 p.

Pourreza, A., Lee, W., Reza, E., Schueller, J., Raveh, E. 2015. An optimum method for real-time infield detection of Huanglongbing disease using a vision sensor. Computers and Electronics in Agriculture. 110, 221-232.

Porep, J.U., Mattes, A., Pour Nikfardjam, M.S., Kammerer, D.R., Carle, R. 2015. Implementation of an on-line near infrared/visible (NIR/VIS) spectrometer for rapid quality assessment of grapes upon receival at wineries. Aust. J. Grape Wine Res. 21, 69-79,
Qin, J., Chao, K., Kim, M. 2011. Investigation of Raman chemical imaging for detection of lycopene changes in tomatoes during postharvest ripening. J. Food Eng. 107, 277-28.

Rubinson, K., Rubinson, J. 2001. Analisis instrumental. Prentice Hall. 872 p.

Sankaran, S., Mishra, A., Ehsani, R., Davis, C. 2010. A review of advanced techniques for detecting plant diseases. Computers and Electronics in Agriculture. 72, 1-13.

Skoog, D., Holler, F., Nieman, T. 2001. Principios de Análisis Instrumental. $5^{\text {ta }}$ Ed. McGraw Hill. 1028 p.

Soler, G., Losada, J., Vargas, C. 2013. Caracterización vibracional por espectroscopía Raman del grano de Café tipo comercial. Scientia et Technica. Año XVIII, Vol. 18, No 1.

Usha, K., Singh, B. 2013. Potential applications of remote sensing in horticulture: A review. Sci Hortic-Amsterdam. 153, 71-83.

Withnall, R., Chowdhry, B., Silver, J., Edwards, H., de Oliveira, L. 2003. Raman spectra of carotenoids in natural products. Spectrochim. Acta Part A 59, 2207-2212. 